\title{
Coarctation of the aorta: difficulties in prenatal diagnosis
}

\author{
Gurleen K Sharland, Kit-Yee Chan, Lindsey D Allan
}

\begin{abstract}
Objective-To formulate echocardiographic criteria for the prenatal diagnosis of coarctation of the aorta.

Design-A retrospective study examining the echocardiograms of fetuses with a verified aortic arch abnormality and those in whom the diagnosis was suspected prenatally but was not subsequently confirmed.
\end{abstract}

Setting-Tertiary referral centre for fetal echocardiography.

Patients-87 fetuses in whom the diagnosis of coarctation was correctly made in 54 , suspected but unproved in 24 , and overlooked prenatally in nine.

Main outcome measures-Measurements of left and right ventricular size, the diameters of the great arteries, the diameters of the left and right atrioventricular valvar orifices, the appearance of the aortic arch, and the direction of the flow of blood across the foramen ovale. Results-Measurements of the ventricular widths, diameters of the great arteries, or the diameters of the atrioventricular valvar orifices, did not allow clear distinction between cases that definitely had a coarctation and those in whom the diagnosis was unproved. The appearance of the aortic arch, particularly in the horizontal projection, was more helpful in distinguishing cases of coarctation, although this also was not always diagnostic. A predominantly left to right shunt across the foramen ovale was detected more often in cases with a substantiated coarctation (58\%) than in those with an unproved diagnosis (12\%).

Conclusions-The most severe forms of coarctation are associated with relative hypoplasia of the left heart structures compared with the right and a correct diagnosis can be made in early pregnancy. The milder forms of coarctation, however, are consistent with a normal early fetal echocardiogram. In late pregnancy it may be impossible to exclude coarctation categorically as the right heart structures may appear larger than the left in the normal fetus. Thus although a combination of echocardiographic features can correctly identify aortic arch anomalies in the fetus, none either alone or in combination, could clearly distinguish between real and false positive cases, particularly in late gestation.

(Br Heart f 1994;71:70-75)
Fetal echocardiography can be used to detect congenital heart disease prenatally with a high degree of accuracy. ${ }^{2}$ Over the past 10 years most varieties of cardiac malformation have been described in the fetus and the natural history of some types documented. ${ }^{34}$ Previous publications from our department have shown that prenatal diagnosis of coarctation of the aorta is possible, ${ }^{5}$ and also that coarctation of the aorta evolves in intrauterine life. ${ }^{6}$ With greater experience, however, it has become evident that the prenatal diagnosis of coarctation of the aorta is more difficult than previously described. Between 1980 and 1990 , although the diagnosis of coarctation of the aorta or interruption of the aortic arch was made correctly in most cases, it became apparent that this diagnosis can be impossible to predict in every case or to exclude definitively. To try to improve the accuracy of this diagnosis before birth, the echocardiograms of all fetuses in whom the diagnosis was made or suspected were examined retrospectively. In this way it was hoped to formulate echocardiographic criteria for the prenatal diagnosis of coarctation of the aorta.

\section{Patients and methods}

Between 1980 and the end of 1990 over 8000 high risk pregnancies were referred for fetal echocardiography. ${ }^{7}$ A structural malformation of the fetal heart was identified in 615 fetuses and subsequently confirmed postnatally or at postmortem examination. Of these 615 cases 54 were correctly diagnosed as having either a coarctation of the aorta or an interrupted aortic arch. Over the same time period there were a further 24 fetuses in whom the diagnosis of coarctation had either been suspected or could not be excluded confidently, but in whom no abnormality of the aortic arch was subsequently confirmed. Over the same 10 year period there were nine cases of coarctation of the aorta detected postnatally where the prenatal echocardiogram had been passed as normal. The study group comprises these 87 fetuses in whom there was either a correct diagnosis of coarctation of the aorta or in whom there had been a false positive or false negative diagnosis.

A videotape recording of the echocardiogram in each case was studied retrospectively. Each fetus was examined with either a Hewlett Packard 77020 phased array system or an Advanced Technology Laboratories Ultramark 4 system. A $5 \mathrm{MHz}$ transducer was used in most cases, but a $3.5 \mathrm{MHz}$ transducer was also available. With a frozen frame from a real time image the diameters of the 
aortic root, main pulmonary artery, mitral and tricuspid valve orifices, and the maximum width of the left and right ventricles were measured and compared with normal reference ranges. ${ }^{8}$ We measured the maximum width of the left and right ventricle in the four chamber projection of the fetal heart just below the atrioventricular valve orifice. We made measurements at the end of diastole when the atrioventricular valves were closed but before the onset of systole. The mitral and tricuspid valves were imaged in the four chamber projection. The valve ring was measured in diastole when the atrioventricular valve was open. We used several projections for measuring the great arteries. In all cases, however, the measurements were made when the walls of the artery were perpendicular in orientation to the ultrasound beam. The internal diameter of the vessel was measured in diastole when the arterial valve was closed. The ratio of the left to right ventricular width, the ratio of the diameters of the mitral to tricuspid valve, and the ratio of the diameters of the aortic root to pulmonary artery were also calculated. A $2 \times 2 \chi^{2}$ test was used to detect any significant difference in the cardiac measurements in the cases that had a coarctation or arch interruption compared with those in whom a false positive diagnosis was made. The aortic arch was subjectively assessed as being small or normal in the cases where transverse views of the aortic arch had been recorded where the size of the arch and duct could be directly compared. Doppler characteristics of blood flow across the aortic and pulmonary valves and the aortic arch that were recorded on videotape were also noted. In more recent cases $(n=20)$, the direction of blood flow across the foramen ovale was examined with colour flow imaging. ${ }^{9}$

In 19 patients, echocardiograms were repeated in later pregnancy. Sequential

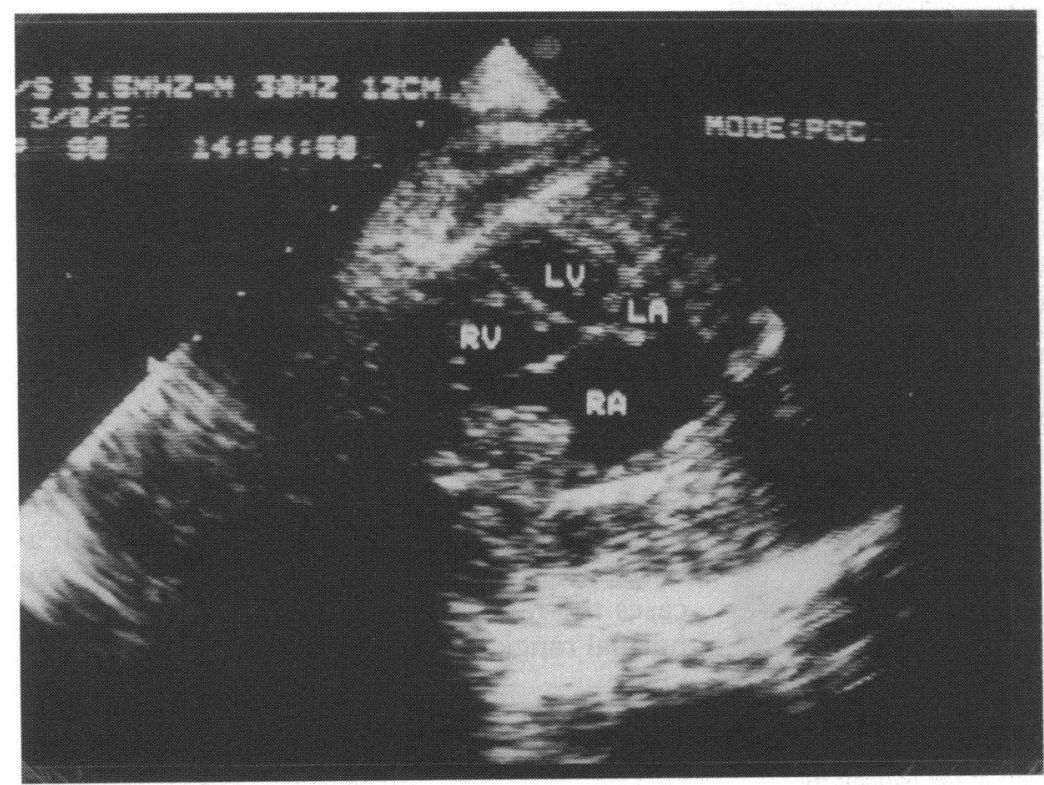

Figure 1 The appearance of the four chamber view in a fetus with coarctation of the aorta. There is considerable disproportion between the sizes of the left and right heart structures. $L A$, left atrium; $L V$, left ventricle, $R A$, right atrium; $R V$, right ventricle. measurements were expressed as a proportion of the expected mean for gestational age so that these could be more easily compared graphically.

\section{Results}

The diagnosis of coarctation of the aorta or interrupted aortic arch was suspected on fetal echocardiography in 78 cases. Of these, 50 fetuses had coarctation, four an interrupted aortic arch, and 24 fetuses were reported to have a normal aortic arch in the neonatal period. There were a further nine fetuses in whom the prenatal echocardiographic features were thought to be normal at the time of the study, but in whom a coarctation was found postnatally. These nine form the false negative group.

The diagnosis was suspected echocardiographically when there was a disparity between the relative sizes of the left and right heart structures. Thus the left ventricle appeared smaller than the right ventricle and the aorta smaller than the pulmonary artery. Figure 1 shows an example of a four chamber view of a fetus with verified coarctation.

\section{GESTATIONAL AGE AT PRESENTATION AND} ACCURACY OF DIAGNOSIS

The median (range) gestational age at presentation of the fetuses that had a verified coarctation was $21(16-40)$ weeks. The median (range) gestational age at which the diagnosis of coarctation was suspected in the false positive group was 29 (18-38) weeks. In most, this was also the age at which the fetus first presented. However, there were four fetuses that presented initially between $18-20$ weeks, when the images were considered normal. In these four cases the diagnosis was suspected on a subsequent study, which in all four cases was performed after 28 weeks of gestation. The diagnosis of coarctation was suspected in 49 fetuses before 24 weeks of gestation. The diagnosis was subsequently confirmed in 42 $(86 \%)$ of these 49 cases. By contrast, after 24 weeks' gestation the diagnosis was suspected in 29 fetuses but proved postnatally in only $12(41 \%)$. Thus a false positive diagnosis was much more likely to be made in later pregnancy. In six of the seven false positive cases in whom the diagnosis was suspected before 24 weeks of gestation, another abnormality was subsequently detected; four fetuses had a chromosomal anomaly, one an isolated ventricular septal defect, and one had the Vater association.

The median (range) gestational age of the nine false negative cases was 18 (17-33) weeks. Except for one case seen at 33 weeks, all the cases in whom the diagnosis had been overlooked prenatally were studied before 20 weeks of gestation.

\section{LEFT VENTRICULAR WIDTH}

Of the cases in whom cardiac measurements were possible, the left ventricular width was below the normal range in $14(32 \%)$ of 44 fetuses that had been correctly diagnosed 
prenatally as having a coarctation, in one $(20 \%)$ of five cases with a false negative diagnosis, and in six $(27 \%)$ of 22 cases with a false positive diagnosis. In the remaining cases the left ventricular width at presentation was within the normal range for the gestational age.

\section{RIGHT VENTRICULAR WIDTH}

Of the cases in whom cardiac measurements were possible the right ventricular width was above the normal range in eight $(18 \%)$ of 44 fetuses that had been correctly diagnosed prenatally as having a coarctation and in nine (41\%) of 22 cases with a false positive diagnosis. In the remaining cases the right ventricular width at presentation was within the normal range for the gestational age.

RATIO OF LEFT TO RIGHT VENTRICULAR WIDTH Of the cases in whom cardiac measurements were possible, the ratio of the left to right ventricular width was below the normal range in $44(90 \%)$ of 49 fetuses that had been correctly diagnosed prenatally as having a coarctation, in two $(33 \%)$ of six cases with a false negative diagnosis, and in $15(65 \%)$ of 23 cases with a false positive diagnosis. In the remaining cases the ratio of the left to right ventricular width at presentation was within the normal range for the gestational age.

\section{DIAMETER OF THE AORTIC ROOT}

Of the cases in whom measurements were possible, the diameter of the aortic root was below the normal range in nine $(20 \%)$ of 46 fetuses that had been correctly diagnosed prenatally as having a coarctation, in one $(20 \%)$ of five cases with a false negative diagnosis, and in five $(23 \%)$ of 22 cases with a false positive diagnosis. In the remaining cases the aortic root diameter at presentation was within the normal range for the gestational age.

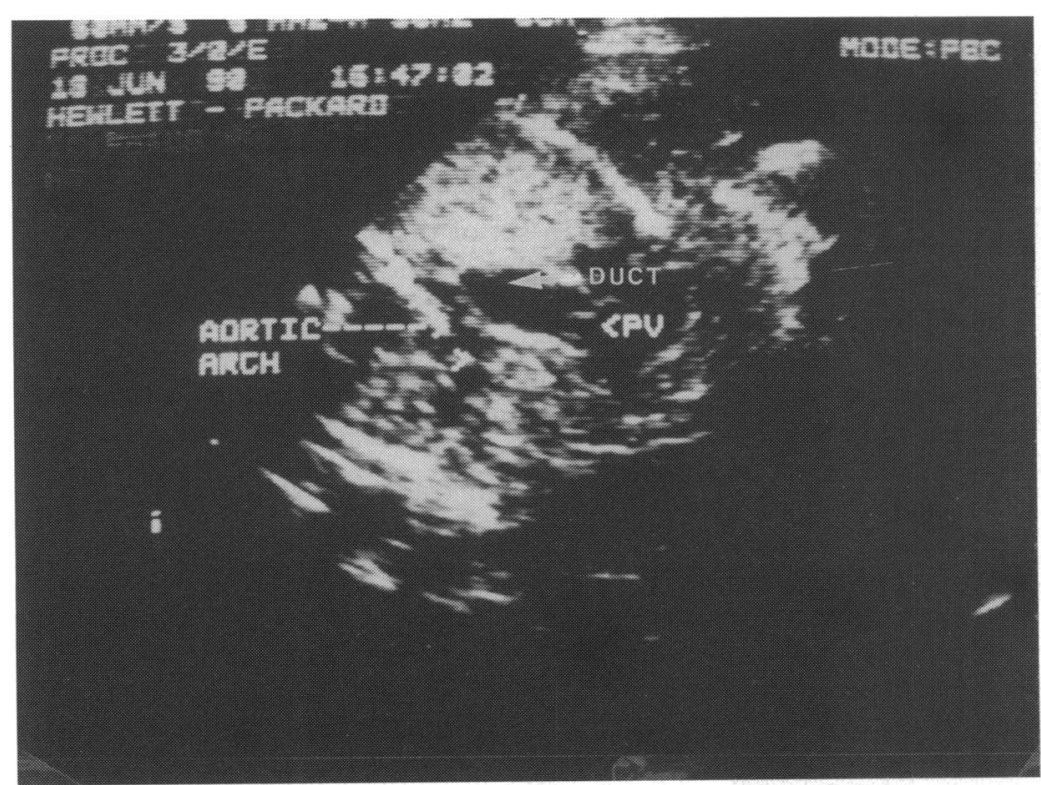

Figure 2 Horizontal section across the fetal thorax showing a view of the pulmonary artery ductal connection and the aortic arch in a fetus with coarctation of the aorta. The aortic arch is hypoplastic and the pulmonary artery and duct appear dilated by comparison. PV, pulmonary valve.
DIAMETER OF THE PULMONARY ARTERY

Of the cases in whom measurements were possible, the diameter of the main pulmonary artery was above the normal range in 10 $(22 \%)$ of 46 fetuses that had been correctly diagnosed prenatally as having a coarctation and in five $(23 \%)$ of 22 cases with a false positive diagnosis. In one of five false negative cases the value was below the fifth centile of the normal range. In the remaining cases the diameter of the pulmonary artery was within the normal range for the gestational age.

\section{RATIO OF AORTA TO PULMONARY ARTERY}

The ratio of the diameter of the aortic root to that of the pulmonary artery at presentation was below the normal range in $37(76 \%)$ of 49 fetuses that had been correctly diagnosed prenatally as having a coarctation, in two $(22 \%)$ of nine cases with a false negative diagnosis, and in $10(42 \%)$ of 24 cases with a false positive diagnosis. In the remaining cases the ratio at presentation was within the normal range for the gestational age.

\section{MITRAL VALVE ORIFICE}

Of the cases in whom measurements were possible the diameter of the mitral valve orifice was below the normal range in $16(39 \%)$ of 41 fetuses that had been correctly diagnosed prenatally as having a coarctation, in one $(33 \%)$ of three cases with a false negative diagnosis, and in $10(50 \%)$ of 20 cases with a false positive diagnosis. In the remaining cases the diameter of the mitral valve orifice at presentation was within the normal range for the gestational age.

\section{TRICUSPID VALVE ORIFICE}

Of the cases in whom measurements were possible the diameter of the tricuspid valve orifice was above the normal range in seven $(16 \%)$ of 45 fetuses that had been correctly diagnosed prenatally as having a coarctation and in six (32\%) of 20 cases with a false positive diagnosis. In three cases that had a coarctation the diameter of the tricuspid valve was smaller than expected for gestational age. In all the remaining cases, the diameter of the tricuspid valve orifice, was within the normal range for the gestational age.

\section{RATIO OF DIAMETER OF MITRAL TO TRICUSPID} VALVE ORIFICE

The ratio of the diameter of the mitral valve orifice to that of the tricuspid valve orifice was below the normal range in $40(93 \%)$ of 43 fetuses that had been correctly diagnosed prenatally as having a coarctation, in one (33\%) of three cases with a false negative diagnosis, and in $17(85 \%)$ of 20 cases with a false positive diagnosis. In the remaining cases the ratio at presentation was within the normal range for the gestational age.

THE $\chi^{2}$ ANALYSIS

A $2 \times 2 \chi^{2}$ test was applied to each group of measurements to see if cases with a coarctation could be distinguished from those without on the basis of the cardiac measurements. 


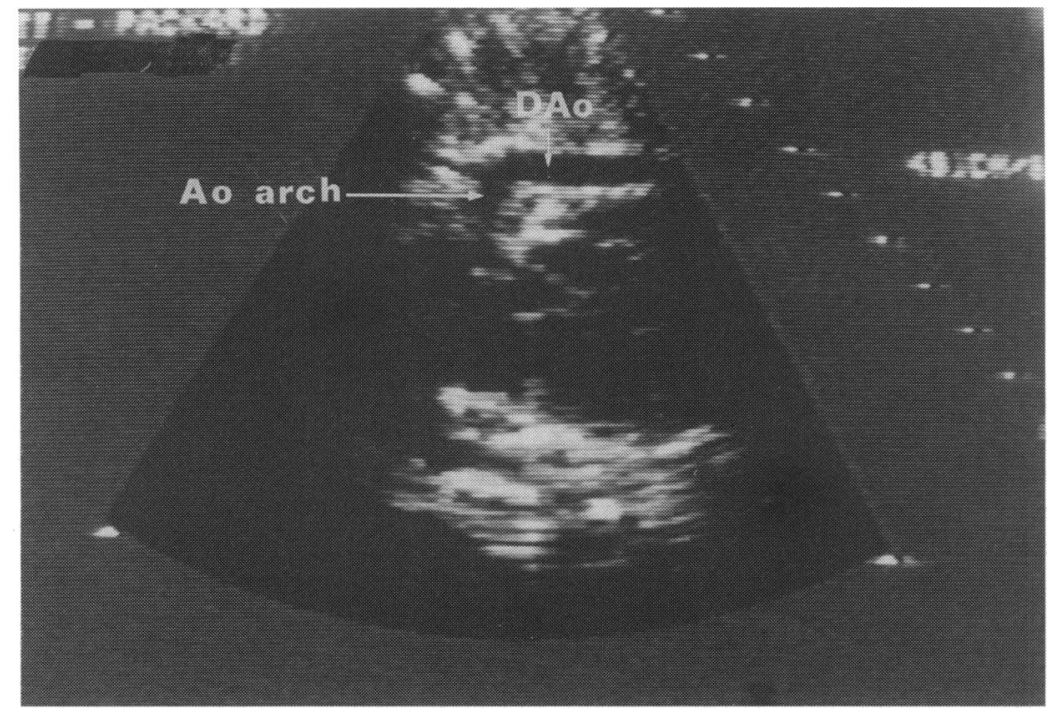

Figure 3 Longitudinal section of the fetus showing a view of the aortic arch. The arch appears normal in this view even though this fetus had a coarctation of the aorta. Ao arch, aortic arch; $D A o$, descending aorta.

This did not reach significance for any of the variables.

\section{APPEARANCE OF THE ARCH}

The aortic arch was classified as small, normal, or not adequately seen at the time of echocardiographic review. The size of the arch was directly compared where possible with the size of the pulmonary artery and duct in a transverse section across the fetal thorax. The size of the two vessels should normally be similar in this projection. Figure 2 shows an example from a fetus with proved coarctation. In this case the transverse arch is hypoplastic compared with the duct. The table summarises the findings of the appearance of the aortic arch. In some fetuses only the longitudinal section of the aortic arch had been recorded on videotape. This was less useful in the diagnosis than the transverse view as the arch appeared normal in some cases in whom the diagnosis was subsequently confirmed (fig 3).

\section{DOPPLER STUDIES}

Although Doppler traces were not available in all cases no abnormal Doppler velocities were found across the aortic or pulmonary valves

Figure 4 Sequential measurements of left ventricular width expressed as a ratio of the normal mean for the gestational age.
Appearance of aortic arch

\begin{tabular}{lccc}
\hline & Small & Normal & Not seen \\
\hline Coarctation & 33 & 7 & 10 \\
Interrupted & & & 4 \\
$\quad$ arch & - & -15 & 4 \\
False positive & 5 & 4 & 3 \\
\hline
\end{tabular}

or in the aortic arch. The direction of blood flow across the foramen ovale was examined with colour flow imaging in 20 cases, 12 of whom had a proved coarctation and eight of whom were false positive cases. The normal shunting across the foramen ovale is predominantly right to left in fetal life. Of the 12 fetuses with a proved coarctation seven had predominantly a left to right shunt across the foramen and five had bidirectional shunting. Of the eight fetuses with a false positive diagnosis six showed bidirectional shunting across the foramen, in one there was predominantly a left to right shunt, and in the remaining case there was predominantly a right to left shunt.

\section{SEQUENTIAL STUDIES}

Sequential studies were performed in 19 fetuses, eight of whom proved to be false positive cases and two were false negative cases. In most cases with a coarctation the left ventricle and the aortic root grew at a reduced rate compared with normal, so that with advancing gestational age, the values as a proportion of the normal mean for the gestational age, became smaller (figs 4 and 5). Sequential measurements of left ventricular width were possible in nine fetuses in whom an arch abnormality had been correctly predicted, one fetus with a false negative diagnosis, and eight fetuses with a false positive diagnosis. The left ventricular width became smaller compared with the mean for gestational age in eight $(80 \%)$ but of 10 fetuses that proved to have an arch abnormality and four (50\%) out of eight fetuses with no arch abnormality. Sequential aortic root measurements were made in nine fetuses in whom an arch abnormality had been correctly

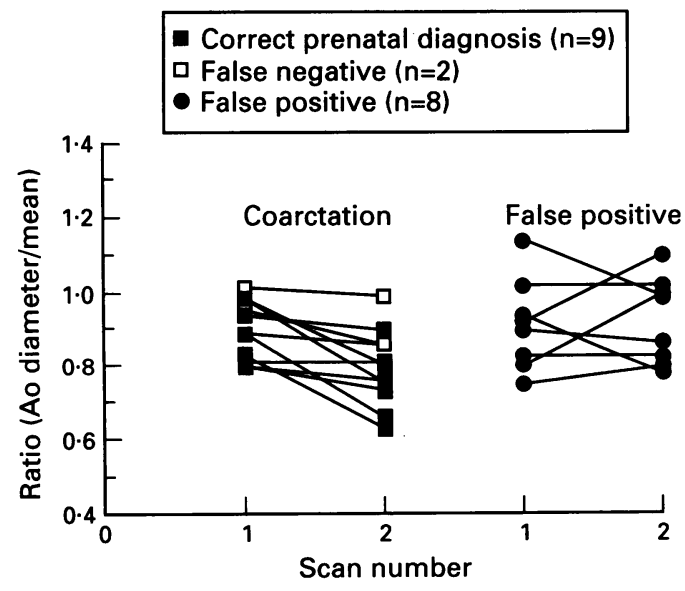

Figure 5 Sequential measurements of aortic root diameter expressed as a ratio of the normal mean for the gestational age. 
predicted, two fetuses with a false negative diagnosis, and eight fetuses with a false positive diagnosis. The diameter of the aortic root became smaller compared with the mean for gestational age in nine $(82 \%)$ out of 11 fetuses that proved to have an arch abnormality and two (25\%) out of eight fetuses with no arch abnormality.

\section{OUTCOME}

Figure 6 summarises the outcome in all 87 cases. Of these 63 cases that had an arch abnormality (54 diagnosed prenatally and nine false negative cases) there are 18 survivors. In 28 cases the parents elected for termination of pregnancy after diagnosis in early pregnancy. Five pregnancies resulted in a spontaneous intrauterine death. Twelve babies died postnatally: eight in the neonatal period and a further four in infancy or childhood. Of these 12 postnatal deaths three had a discrete coarctation lesion, three had a hypoplastic aortic arch, one case had an interrupted aortic arch, two were associated with a ventricular septal defect, and three with multiple congenital anomalies. Of the 54 cases that had been correctly diagnosed prenatally as having an arch abnormality there were 26 continuing pregnancies. Of these 26 there are 10 survivors giving a mortality of $62 \%$ for this condition diagnosed prenatally. Of the nine false negative cases one baby died in the neonatal period and there are eight survivors.

A false positive diagnosis was made in 24 cases of whom there are 16 survivors. Termination of pregnancy took place in four cases three of whom had an associated chromosomal anomaly. In the fourth case ventricular disproportion was detected in early pregnancy and the diagnosis of coarctation was suspected. It was planned to rescan but the mother elected to interrupt the pregnancy against advice. Postmortem examination showed hypoplasia of the ascending aorta but no discrete coarctation. This case has been counted as a false positive. One fetus with tri-

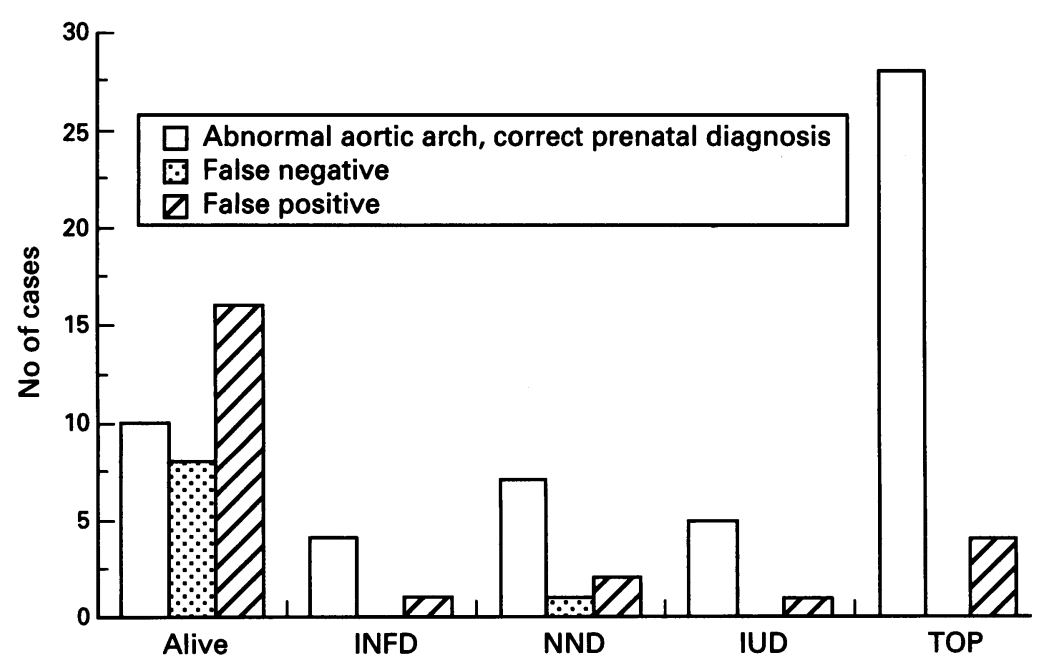

Figure 6 Outcome of pregnancy in 87 fetuses with either an abnormality of the aortic arch or a false positive diagnosis of an abnormality. INFD, death in infancy or childhood; NND, neonatal death; IUD, spontaneous intrauterine death; TOP, termination of pregnancy. somy 18 had spontaneous intrauterine death and three babies died postnatally-one of persistent fetal circulation, one with trisomy 18 , and the third of multiple congenital anomalies including a ventricular septal defect.

\section{Discussion}

Coarctation of the aorta can be diagnosed prenatally from 18 weeks of gestation onwards. ${ }^{5}$ The diagnosis is suspected when there is a disparity between the relative sizes of the left and right heart structures-that is, the aorta is smaller than the pulmonary artery and the left ventricle is smaller than the right ventricle. This study shows, however, that these findings can also be detected in some fetuses in whom there is no evidence postnatally of a coarctation. The ratios of left/right ventricular width, diameters of aortic root pulmonary artery and mitral/tricuspid valve orifice were reduced compared with the normal range in most cases thus confirming the echocardiographic appearance of ventricular disproportion and a disparity in the sizes of the two great arteries. These ratios did not, however, help to distinguish cases of coarctation from those with a false positive diagnosis. The reason the diagnosis of an aortic arch abnormality was suspected in the false positive cases is that they showed the echocardiographic features that are associated with prenatal coarctation of the aorta. Furthermore in some cases, particularly in early pregnancy, the relative sizes of the left and right sides of the heart may seem normal despite the detection postnatally of a coarctation lesion.

The disparity that occurs between the two sides of the heart has been reported to be due to dilatation of the right heart structures. ${ }^{10}$ The results of this study show that although the right ventricular width and the diameters of the tricuspid valve orifice and pulmonary artery were enlarged compared with the normal ranges in some fetuses, in most the measurements were within the normal range at the time of presentation. Morrow et al suggested that the recognition of arch hypoplasia and increased diameters of the pulmonary valve and pulmonary artery in the fetus may enhance prenatal detection of coarctation. ${ }^{11}$ Clearly this is not the case from our study, as although the pulmonary artery appears relatively dilated compared with the arch in cases of fetal coarctation, actual measurements compared with normal ranges are within normal limits in many cases. Measurements of the ventricular widths, diameters of the great arteries, or the diameters of the atrioventricular valvar orifices, did not allow distinction between cases that definitely had a coarctation and those in whom the diagnosis was not proved. The left ventricular width and aortic root diameter were found to be below normal values for the gestational age in some cases, but were within the normal range in most at presentation. Sequential measurements of these two values showed a decrease in rate of 
growth in some cases, such that the value decreased compared with the mean for the gestational age. This was particularly true for the aortic root diameter, which became relatively smaller in most cases with a proved coarctation, but growth was not reduced in most of the false positive cases. Sequential studies may therefore be helpful in making a more confident diagnosis.

The appearance of the aortic arch was more helpful in distinguishing cases of coarctation, although this also was not definitive. The arch was adequately imaged in 40 cases that had a coarctation and was considered to be small in $33(83 \%)$. The arch was not seen in all four cases that proved to have an arch interruption. In 15 of the 20 false positive cases $(75 \%)$, in whom an arch was adequately imaged, the arch was considered normal. In the six false negative cases in whom the arch was adequately imaged, the appearance was normal at initial presentation.

Doppler studies of flow across arterial valves did not seem to be helpful in distinguishing cases from false positives. Examination of transatrial flow across the foramen ovale may be helpful in the diagnosis of coarctation. Feit et al 1991 reported that seven of nine fetuses with left heart obstruction studied with colour Doppler showed reversal of the normal flow pattern across the foramen with unidirectional left to right transforamenal flow. ${ }^{12}$ The findings of our study support this, although this was not universally found in all abnormal cases, and may occasionally be detected in the absence of left heart obstruction. The presence of a predominantly left to right shunt across the foramen ovale in conjunction with other echocardiographic features of coarctation, may assist in making a more accurate diagnosis.

The results indicate that the most severe forms of coarctation are associated with relative hypoplasia of the left heart structures compared with the right in early pregnancy and that a correct diagnosis can be made. There is no definitive sign but combining all the variables of assessment will detect most cases. The milder forms of coarctation are consistent with a normal early fetal echocardiogram and some of these cases may only become evident after the duct closes postnatally. This may have to be accepted as a limitation of prenatal diagnosis. The false positive diagnoses are of greater concern. In most $(71 \%)$, the diagnosis was suspected after 24 weeks of gestation. In later pregnancy the right ventricle can be larger than the left in the normal fetus, ${ }^{8}$ and examination of the other features may not always discriminate between the real and the false positive case.

When all the echocardiographic features of coarctation are present in early pregnancy the diagnosis is highly likely to be correct and the outlook for postnatal survival has been poor. The mortality in this series is $64 \%$ in continuing pregnancies where the diagnosis was made before a gestational age of 24 weeks. This may lead parents to consider termination of pregnancy but it is important that they understand that this can be an insecure diagnosis especially if the echocardiographic findings are subtle. In these circumstances, the growth of the cardiac chambers should be followed up and compared with normal values for the gestational age. Meanwhile the rest of the fetus should be examined and chromosomal analysis obtained. ${ }^{13}{ }^{14}$ Although coarctation is commonly known to occur in association with the $45 \mathrm{X} 0$ karyotype of Turner's syndrome, trisomies 18 and 13 also occur. Also, this study shows that it is important to look for a karyotypic abnormality in cases where there is a disparity in the two sides of the heart even in the absence of a coarctation lesion. In late pregnancy, it may be impossible to exclude coarctation categorically. Our policy, in these cases where coarctation seems a possible diagnosis, is to deliver in our centre to avoid the possible necessity of transferring a sick neonate from another hospital after the symptoms of coarctation have developed. The diagnosis of coarctation cannot be excluded postnatally until the duct has closed and there are no abnormal physical signs evident.

In summary, although a combination of echocardiographic features can correctly identify most anomalies of the aortic arch in the fetus, none, either alone or in combination, clearly distinguished between real and false positive cases, particularly in late gestation.

1 Kleinman CS, Hobbins JC, Jaffe CC, Lynch DC, Talner NS. Echocardiographic studies of the human fetus: prenatal diagnosis of congenital heart disease and cardiac dysrhythmias. Pediatrics 1980;65:1059-68.

2 Allan LD, Crawford DC, Anderson RH, Tynan MJ. Echocardiographic and anatomical correlations in fetal Echocardiographic and anatomical correlations in

3 Sharland GK, Chita SK, Allan LD. Tricuspid valve dysplasia or displacement in intrauterine life. $f \mathrm{Am}$ Coll plasia or displacement

4 Allan LD, Sharland GK, Tynan M. Natural history of the hypoplastic left heart syndrome. Int $\mathcal{f}$ Cardiol 1989;25: 341-3

5 Allan LD, Chita SK, Anderson RH, Fagg N, Crawford DC, Tynan MJ. Coarctation of the aorta in prenatal life: an echocardiographic, anatomical, and functional study. Br Heart f 1988;59:356-60.

6 Allan LD, Crawford DC, Tynan M. Evolution of coarctation of the aorta in intrauterine life. Br Heart $\mathcal{F} 1984$; 52:471-3.

7 Sharland G, Allan L. Detection of congenital abnormalities of the cardiovascular system by ultrasound. In Chamberlain G, ed. Modern antenatal care of the fetus. Oxford: Blackwell Scientific Publications, 1990:356.

8 Sharland GK, Allan LD. Normal fetal cardiac measurements derived by cross-sectional echocardiography.

Ultrasound in Obstetrics and Gynecology 1992;2:175-81. Doppler in fetal echocardiography. Int $₹$ Cardiol 1990;
Dorland GK, Chita SK, Allan LD. The use of colour 28:229-36.

10 Beekman RH, Rocchini AP. Coarctation of the aorta and interruption of the aortic arch. In: Moller JH, Neal WA interruption of the aortic arch. In: Moller $\mathrm{JH}$, Neal WA, eds. Fetal, neonatal and infant cardiac diac
Connecticut: Appleton and Lange, 1990:497-521.

11 Morrow WR, Huhta JC, Murphy DJ, McNamara DG. Quantitative morphology of the aortic arch in neonatal coarctation. F Am Coll Cardiol 1986;8:616-20.

12 Feit LR, Copel JA, Kleinman CS. Foramen ovale size in the normal and abnormal human fetal heart: an indicator of transatrial flow physiology. Ultrasound in Obstetrics and Gynecology 1991;1:313-9.

13 Allan LD, Sharland GK, Chita SK, Lockhart S, Maxwell DJ. Chromosomal anomalies in fetal congenital heart disease. Ultrasound in Obstetrics and Gynecology 1991;1: 8-11.

14 Copel JA, Cullen M, Green J, Mahoney MJ, Hobbins JC Kleinman CS. The frequency of aneuploidy in prenatally diagnosed congenital heart disease: an indication for fetal karyotyping. Am $\mathcal{f}$ Obstet Gynecol 1988;158 409-13. 